\title{
Electrocatalytic Activity of Individual Pt Nanoparticles Studied by Nanoscale Scanning Electrochemical Microscopy
}

Jiyeon Kim, Christophe Renault, Nikoloz Nioradze, Netzahualcóyotl Arroyo-Currás, Kevin C. Leonard, and Allen J. Bard

\section{Supporting Information}

Table of Contents

(1) A Stable Nanogap in SECM. (Figure S1)-----------------------------------------------------------S2

(2) Artifact of NP Shapes in the SECM Image and the Theoretical Validation of their Geometry

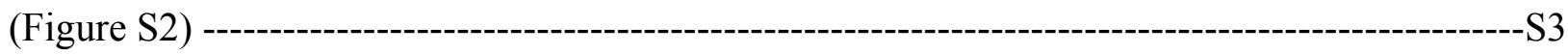

(3) Tip Drift in Lateral Direction and Its Suppression. ---------------------------------------------- S5

(4) Analysis of SECM approach curve for HOR over HOPG surface. (Figure S3, S4) ----------S5

(5) Analysis of Tip Voltammogram for $\mathrm{H}^{+}$reduction/ HOR. (Figure S5) -------------------------S9

(6) The Voltammogram of HOPG Substrate in $\mathrm{HClO}_{4}$ Solution. (Figure S6) --------------------S10 


\section{(1) A Stable Nanogap in SECM.}

To stabilize the nanometer size gap between the tip and substrate during SECM measurement, we conducted SECM experiments in an isothermal chamber, where the thermal drift is suppressed as reported elsewhere. ${ }^{\text {S1 }}$ Also, the use of lockable differential micropositioners (Lockable differential micropositioner DM-25L and DM-13L, Newport) mounted on the SECM stage for long-distance translocation in $\mathrm{x}, \mathrm{y}$ and $\mathrm{z}$ axis and for the tilt adjustment of the substrate stage at the triangular position minimize the physical creeping or drift in SECM system once they are locked.

Approaching the tip over the substrate, then stopping the tip approach at the distance comparable to tip radius and monitoring the tip current over time in $1 \mathrm{mM}$ ferrocenyl methyl trimethylammonium solution as shown in Figure S1 experimentally confirmed the stabilized gap between the tip and substrate. Pt tip with $580 \mathrm{~nm}$ radius was held at $360 \mathrm{~nm}$ above the insulating substrate and tip current was monitored over time, which is a measure of distance between the tip and substrate. In Figure S1, the resulting distance - time curve was shown converted from current - time curve. The tip drift level in $\mathrm{z}$ axis was less than $0.5 \mathrm{~nm} / \mathrm{min}$, which is stable enough to get the high quality SECM image as well as quantitative SECM measurement. 


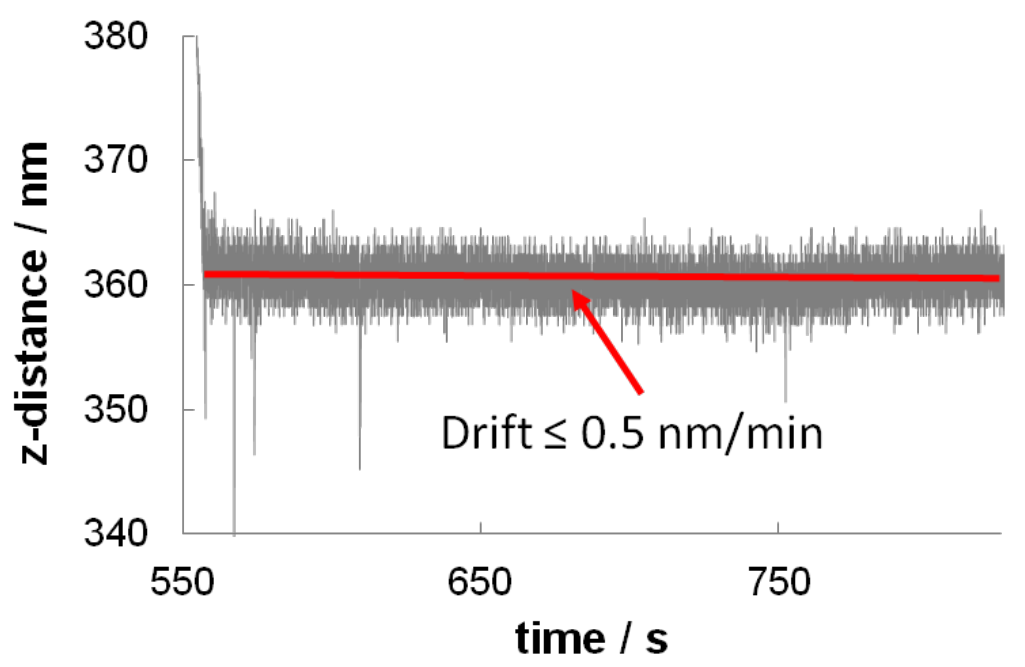

Figure S1. Distance-time curves obtained with $580 \mathrm{~nm}$ radius $\mathrm{Pt}$ tip and $\mathrm{SiO}_{2}$ substrate in $1 \mathrm{mM}$ $\mathrm{FcTMA}^{+}$solution using SECM instruments. The resulting tip position in z-axis is drifted less than $0.5 \mathrm{~nm} / \mathrm{min}$.

\section{(2) Artifact of NP Shapes in the SECM Image and the Theoretical Validation of their}

\section{Geometry.}

Despite good agreement in current magnitudes between theoretical and experimental current profiles over Pt NPs for HOR study under diffusion controlled condition, we observed a big discrepancy in current peak width. Due to the elongated shapes of Pt NPs in SECM images, an ellipsoidal geometry was initially considered. Such a consideration, however, is not reasonable based on the theoretical model of NP nucleation and growth as well as our SEM observation. In fact, the dimensions of NPs with ellipsoidal geometry showed a good fit for cross sectional current profiles in SECM image studied with FcTMA $^{+}$(data not shown).

Ellipsoidal NPs with dimensions of (63 nm, $16 \mathrm{~nm}, 120 \mathrm{~nm})$ and $(108 \mathrm{~nm}, 36 \mathrm{~nm}, 120$ $\mathrm{nm}$ ) as long axis radius, short axis radius, and height in order could give comparable results with hemispherical to nearly spherical NPs with 63 and $120 \mathrm{~nm}$ radii with $120 \mathrm{~nm}$ height in 
topographical analysis. As shown in Figure S2, we theoretically predicted tip current-distance curves over the different shaped NPs as a function of the gap between the tip and NP. The theoretical currents over an individual NP with each dimension and given geometry are presented in the case of a mass transfer limit. At a $90 \mathrm{~nm}$ gap between the tip and NPs for SECM study with $\mathrm{FcTMA}^{+}$, the theoretically predicted currents over each NP under the mass transfer limit are nearly similar between ellipsoidal and hemispherical to spherical geometries. As the tip approaches closer to NPs, the difference in tip currents between different geometries of NPs becomes more obvious. At $14 \mathrm{~nm}$ gap for SECM study for the HOR, each NP with ellipsoidal geometry displays obviously smaller currents than hemispherical $\sim$ spherical shaped NPs, thus given ellipsoidal geometries could not explain a high activity of Pt NPs on the HOR. In that sense, it is likely that the ellipsoidal shaped Pt NP in SECM images could be an artifact.

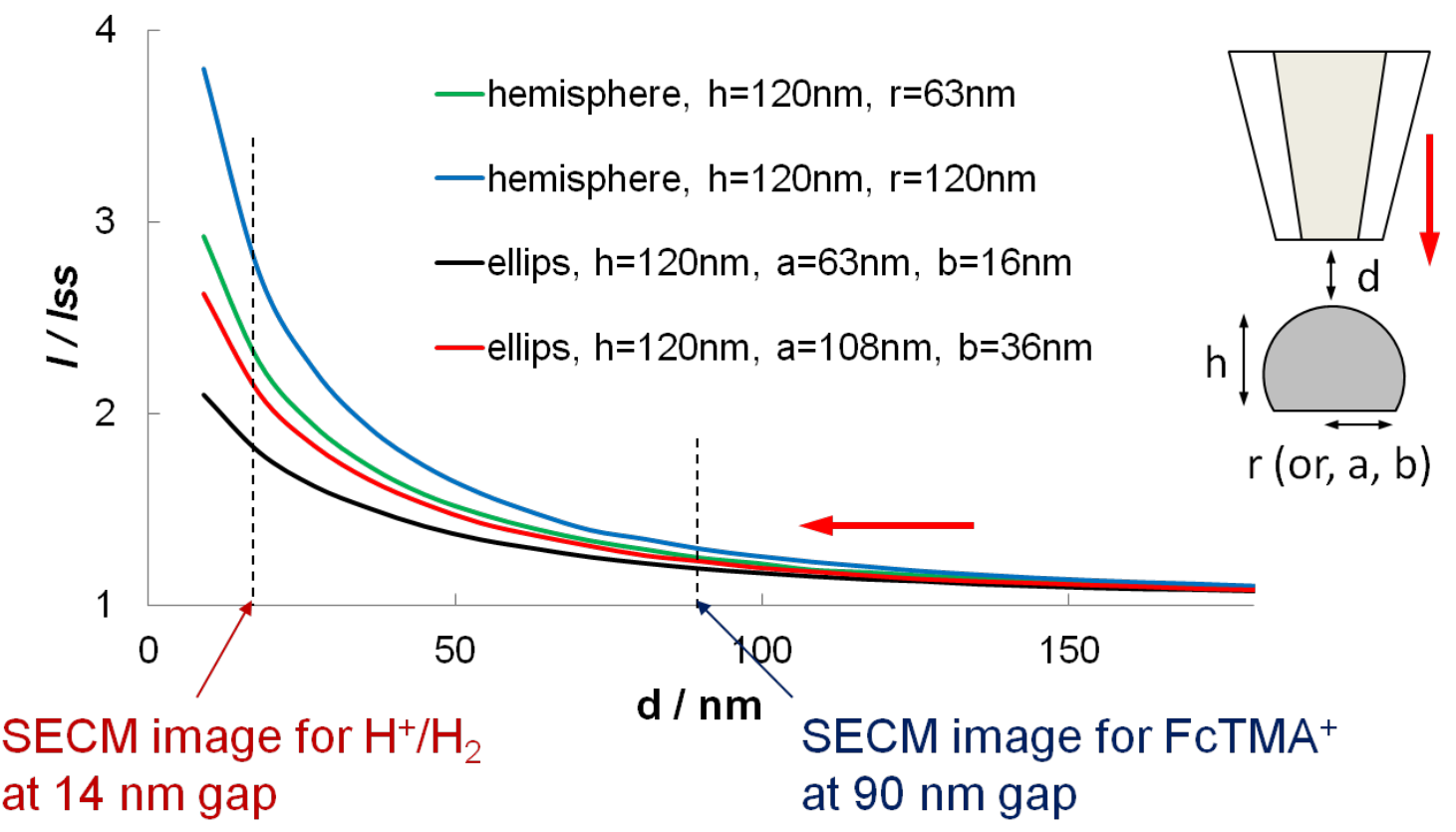

Figure S2. Theoretical current-distance curves for an inlaid-disk tip with RG 2.5 approaching a reactive ellipsoidal, or hemispherical $\sim$ spherical NP with dimensions, $h(=$ height), a ( $=$ long axis radius), $\mathrm{b}$ ( = short axis radius) or $r$ (= radius), on the inert substrate. $d$ represents the gap between the tip and apex of NP. The current is normalized against the limiting current in the bulk 
solution. The resulting tip current over NP is governed by the diffusion control in this analysis. In the right inset, the red arrows denote approaching direction of a tip over a NP.

\section{(3) Tip Drift in Lateral Direction and Its Suppression.}

We speculate that the artifact in SECM image could be attributed to the tip drift in lateral direction during scanning the tip. In our initial instrumentation, a lockable micropositioner was used only in the z-axis. In fact, the lockable micropositioner greatly helped to stabilize the tip position in the $\mathrm{z}$ axis in the isothermal chamber, where the thermal drift was suppressed. As a result, each SECM image showed uniform background current implying no discernible tip drift in the $\mathrm{z}$ axis. In this regard, general micropositioners without locks used in the $\mathrm{x}$ and $\mathrm{y}$ axis could cause a serious drift in the lateral tip position while the tip was scanned by piezo positioner, thereby the SECM image could be distorted in lateral direction. Such distorted images, also can be seen in the other SECM work, where unprecedentedly high spatial resolution images were

introduced. $^{\mathrm{S} 2}$ Based on this speculation, all micropositioners were replaced with lockable micromanipulators. At the same time, the spherical shaped Pt NP was observed in the SECM image studied with $\mathrm{FcTMA}^{+}$as presented in Figure 11, indicating that unwanted tip drifts in lateral direction could be successfully suppressed.

\section{(4) Analysis of SECM approach curve for HOR over HOPG surface.}

We analyze SECM approach curve over HOPG substrate for HOR reaction using the finite element simulation with COMSOL MULTIPHYSICS $\mathrm{v}$ 4.3b. During the direct electrodeposition of Pt NPs on HOPG surface, small Pt "nuclei” were formed on HOPG surface. As a result, the SECM approach curve over the HOPG surface not over Pt NP showed a distinct 
curve attributed to a feedback response from small Pt nuclei for hydrogen oxidation reaction instead of purely inert HOPG surface. 3-D simulation was carried out to analyze the obtained SECM approach curve. Figure S3a, b present the model in 3-D simulation, where Pt tip with RG 2 (the ratio of the radius of the glass sheath with respect to the Pt tip radius) approaches over the array of small Pt nuclei. Additional number of Pt nuclei under the glass sheath outside the Pt tip surface negligibly influences the obtained feedback response, thus we only consider the minimum number of Pt nuclei under Pt tip here (data not shown). Using this 3-D simulation, we numerically solved 3-D steady state diffusion problem for the vertical tip displacement over the Pt nuclei. The diffusion of $\mathrm{H}^{+}$was defined by

$$
\frac{\partial c(x, y, z)}{\partial t}=D_{H^{+}}\left[\frac{\partial^{2} c(x, y, z)}{\partial x^{2}}+\frac{\partial^{2} c(x, y, z)}{\partial y^{2}}+\frac{\partial^{2} c(x, y, z)}{\partial z^{2}}\right]
$$

where $\mathrm{x}, \mathrm{y}$ and $\mathrm{z}$ are the coordinates in directions normal and parallel to the Pt tip length, $c(x, y, z)$ is the concentration of $\mathrm{H}^{+}$and $D_{H}{ }^{+}$is the diffusion coefficient. The dimensionless variables are used in this simulation as

$$
\begin{aligned}
& H=h / a \\
& W=w / a \\
& C(X, Y, Z)=c(x, y, z) / c^{*} \\
& D=d / a \\
& R G=r_{g} / a \\
& R p=r_{p} / a
\end{aligned}
$$


where $c^{*}$ is the bulk concentration, $a$ is the Pt tip radius, $r_{g}$ is the glass sheath radius, $w$ is the simulation space limit in lateral direction, $h$ is the simulation space limit in $\mathrm{z}$ coordinate, $r_{p}$ is the radius of Pt nucleus, and $d$ is the distance from Pt nuclei to the Pt tip surface. Here, the tip and $\mathrm{Pt}$ nucleus reaction were limited by the diffusion of $\mathrm{H}^{+}$or $\mathrm{H}$, respectively.

The boundary conditions are as below.

At the Pt tip surface, $C=0 ; 0 \leq|\mathrm{X}| \leq 1,0 \leq|\mathrm{Y}| \leq 1, \mathrm{Z}=\mathrm{D}$

At Pt nuclei, $\mathrm{C}=1$

At glass sheath, $\frac{\partial C(X, Y, Z)}{\partial n}=0 ; 1<|\mathrm{X}| \leq \mathrm{RG}, 1<|\mathrm{Y}| \leq \mathrm{RG}, \mathrm{D} \leq \mathrm{Z} \leq \mathrm{H}-\mathrm{D}$

At inert HOPG surface, $\frac{\partial C(X, Y, Z)}{\partial n}=0 ; \mathrm{Z}=0,0 \leq|\mathrm{X}| \leq \mathrm{W}, 0 \leq|\mathrm{Y}| \leq \mathrm{W}$

The dimensionless tip current is obtained by integrating the dimensionless diffusion flux over the Pt tip surface as

$$
I_{T}=\frac{i}{i_{s S}}=\frac{\pi}{2} \int_{0}^{1} R\left[\frac{\partial C(X, Y, Z)}{\partial Z}\right] d R
$$

where $i_{s s}=4 x n F D c^{*} a$

$x$ is RG coefficient ( $x=1.10$ for RG 2 tip), $n$ is the number of transferred electron, $F$ is Faraday constant and $D$ is the diffusion coefficient of redox species.

Resultantly, we obtained a good agreement between the experimental approach curve and the theoretically simulated curve when the coverage of small hemispherical Pt nuclei with radius and height c.a. $3 \mathrm{~nm}$ is about $1.1 \%$ under the tip surface as a minimum number of Pt nuclei, where 13 nuclei array with separation distance about 10 times larger than their radius, thus negligible overlap between Pt nuclei is assumed for HOR (Figure S3c). Based on this theoretical analysis, the accurate tip position over the HOPG substrate was determined, which is crucial for the quantitative analysis. 
In addition, we also simulated that the feedback current response at the Pt nanotip over Pt NP is not affected by the presence of Pt nuclei as shown in Figure S4. We predicted currentdistance curves over an individual Pt NP with and without Pt nuclei, where the electron transfer reaction at the Pt NP is controlled by diffusion. Two curves were almost identical. This result simplified all other numerical simulations with multi Pt NPs for the current-later distance profiles, where Pt NPs were only considered to construct the model in 3D.

(a)

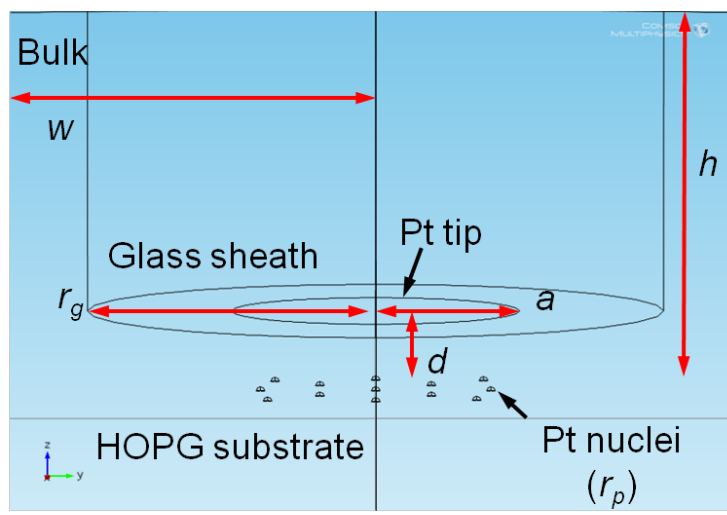

(c)

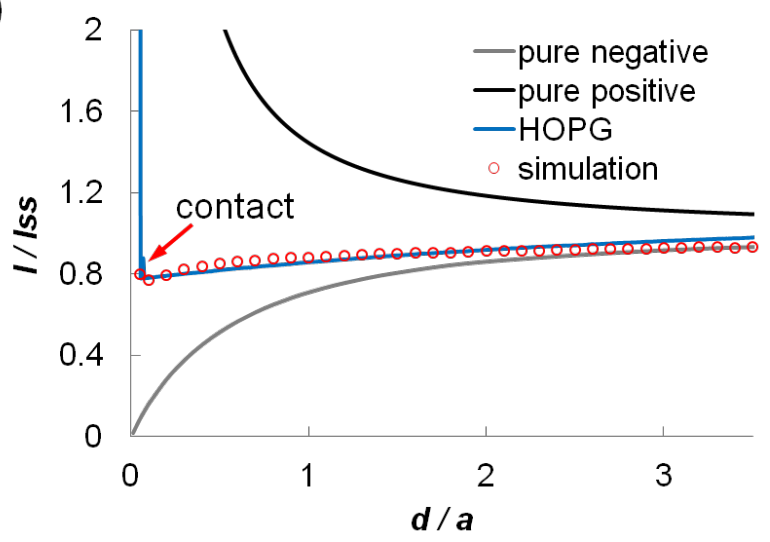

(b)

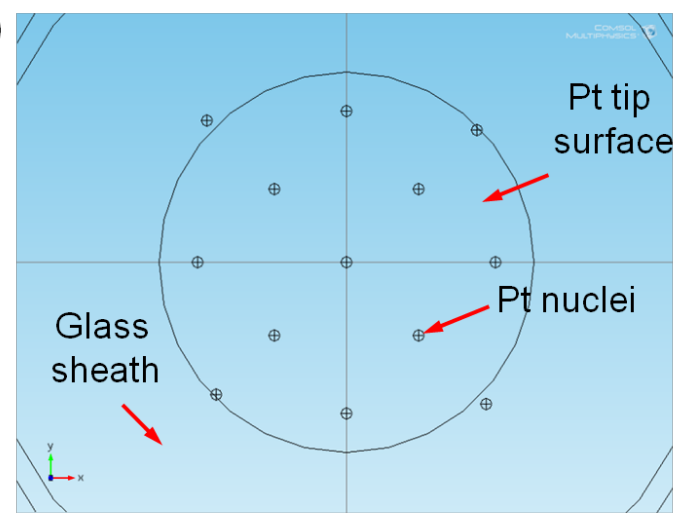

Figure S3. (a, b) Geometries of the 3-D simulation space and dimensions defining the diffusion problem. 13 small Pt nuclei with normalized radius 0.03 with respect to the Pt tip radius are arrayed with a separation distance of $\sim 10$ times of nucleus radius under the tip surface $(\sim 1.1 \%$ coverage under the $\mathrm{Pt}$ tip). a panel is side view and $\mathrm{b}$ panel is top view of 3-D simulation space.

(c) Current-distance curves simulated (open circles) for an inlaid disk Pt tip approaching small Pt nuclei immobilized on the inert HOPG surface in HOR reaction. Simulated curve is in a good fit in experimental curve (blue solid line). 


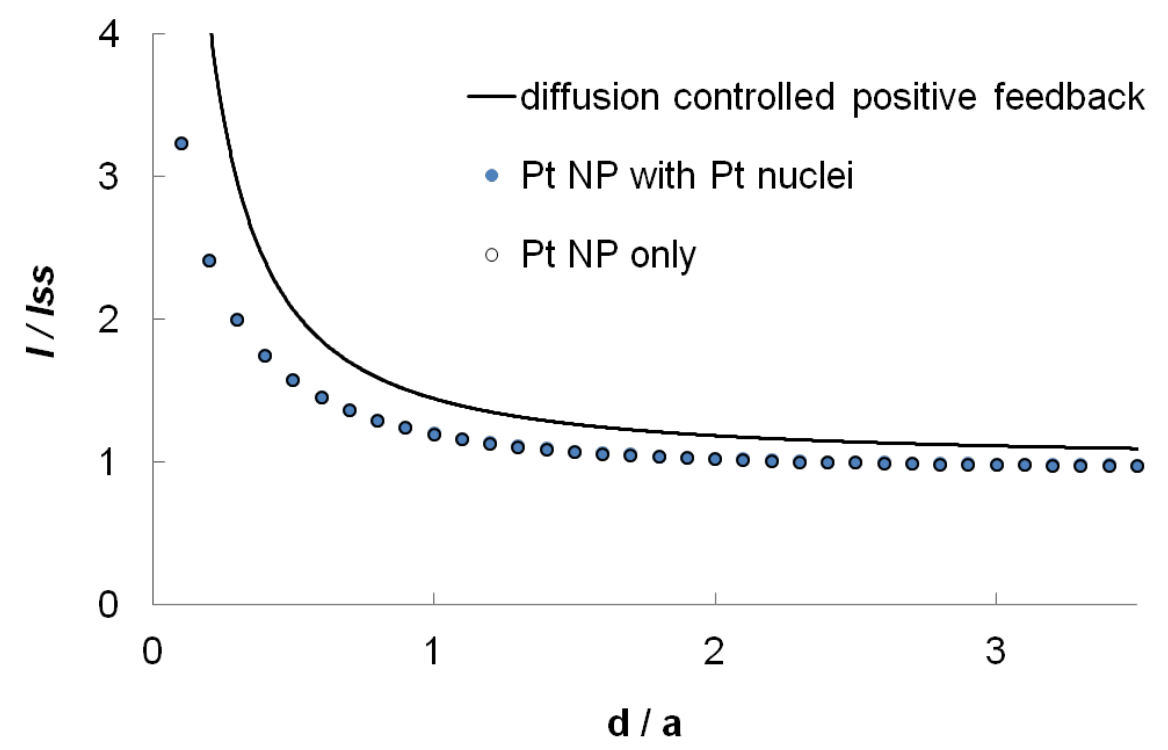

Figure S4. Current-distance curves simulated for an inlaid disk Pt tip approaching small Pt nuclei immobilized on the inert HOPG surface in HOR reaction with (closed circles) or without Pt nuclei (open circles).

\section{(5) Analysis of Tip Voltammogram for $\mathrm{H}^{+}$reduction/ HOR.}

We analyzed our voltammogram obtained with a Pt nanotip in $2 \mathrm{mM} \mathrm{HClO}_{4}, 10 \mathrm{mM}$ $\mathrm{NaClO}_{4}$ solution. The resulting voltammograms corresponds to $\mathrm{H}^{+}$reduction $/ \mathrm{H}_{2}$ oxidation. The reverse wave among this experimental curve was fitted with the theoretical curve with $k_{\text {eff }}^{0}=2$ $\mathrm{cm} / \mathrm{s}\left(\alpha=0.5\right.$ and $\left.E^{0^{\prime}}=-0.69 \mathrm{~V}\right)$ determined as a lower limit of the heterogeneous effective rate constant for HOR. 


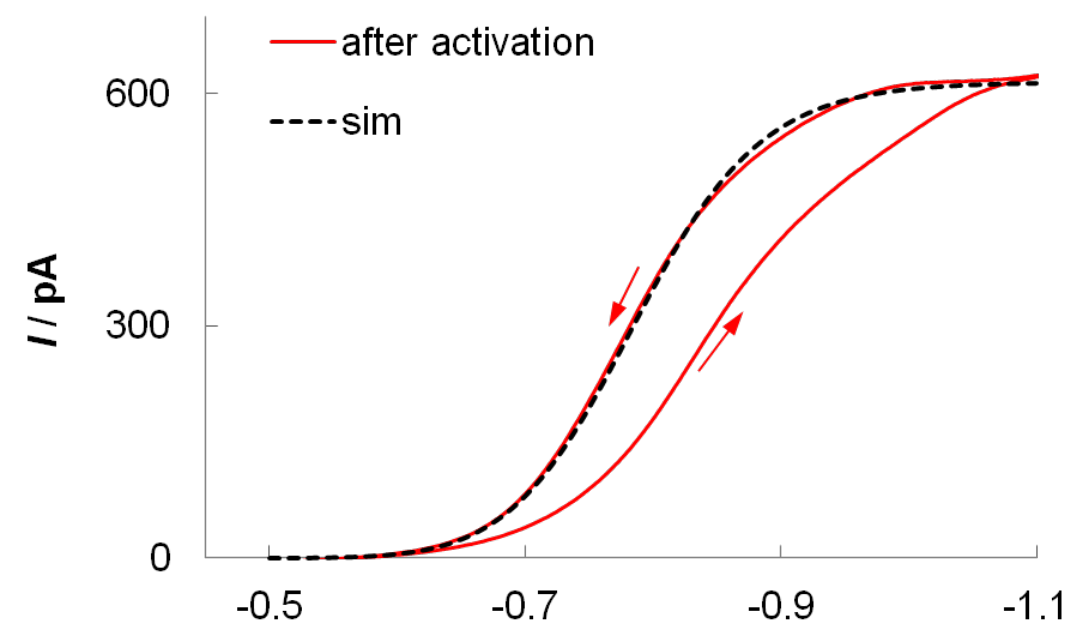

$E / V$ vs. Pt QRE

Figure S5. The voltammogram of a Pt nanotip for $\mathrm{H}^{+}$reduction/ $\mathrm{H}_{2}$ oxidation in $2 \mathrm{mM} \mathrm{HClO}_{4}, 10$ $\mathrm{mM} \mathrm{NaClO}_{4}$ solution at scan rate $20 \mathrm{mV} / \mathrm{s}$. The reverse wave of the experimental curve (red solid line) was fitted with the theoretical curve (dotted line) with obtained kinetic parameters, $k_{\text {eff }}^{0}=2 \mathrm{~cm} / \mathrm{s}\left(\alpha=0.5 E^{0^{\prime}}=-0.69 \mathrm{~V}\right)$.

(6) The Voltammogram of HOPG Substrate in $\mathrm{HClO}_{4}$ Solution.

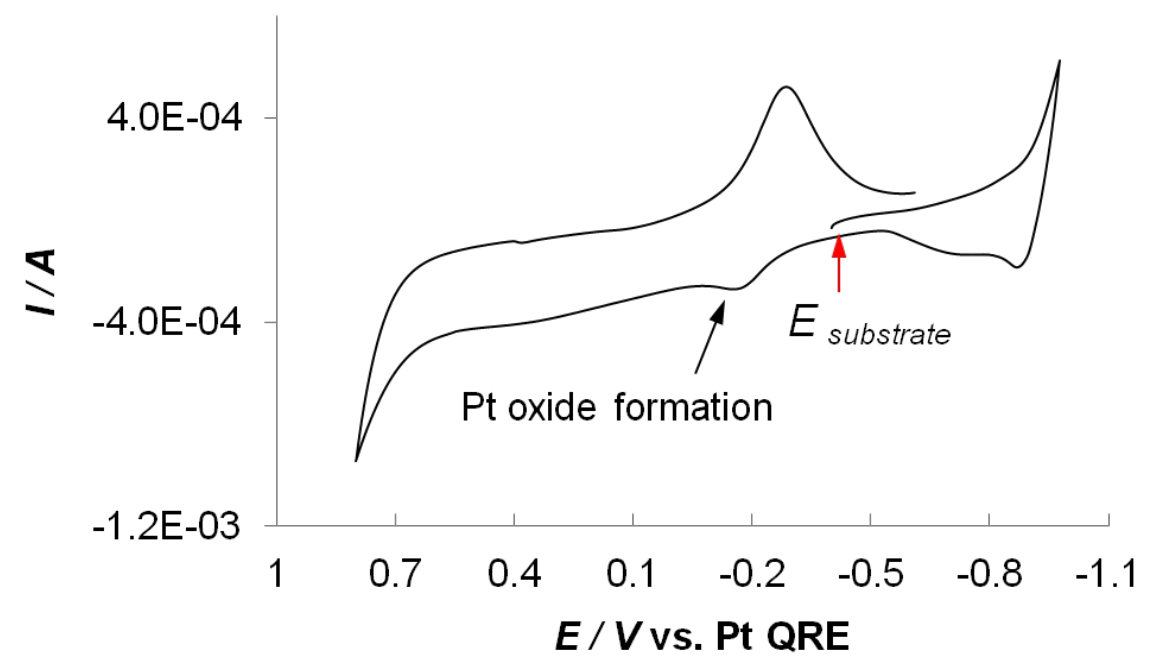

Figure S6. The voltammogram of Pt NPs/HOPG in $2 \mathrm{mM} \mathrm{HClO}_{4}, 10 \mathrm{mM} \mathrm{NaClO}_{4}$ solution at scan rate $100 \mathrm{mV} / \mathrm{s}$. The red arrow denotes the potential applied to Pt NPs/HOPG during SECM imaging. 


\section{References}

S1. Kim, J.; Shen, M.; Nioradze, N.; Amemiya, S., Anal. Chem., 2012, 84, 3489-3492.

S2. Sun, T.; Yu, Y.; Zacher, B. J.; Mirkin, M. V., Angew. Chem. Int. Ed., 2014, 53, 1412014123. 